KIMESIS. 2(2):175-194/JUL-DEZ/1986.

\title{
A INFLUENCIA DA FADIGA mUSCULAR DE MEMBROS SUPERIORES NA ESTABILIDADE MANUAL
}

THE INFLUENCE OF MUSCULAR FATIGUE OF THE ARMS UPON

MANUAL STABILITY

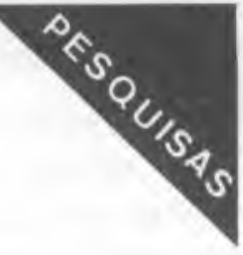

\footnotetext{
* Juarez Vieira do nascimento

* Adair da silva lopes

* Alberto Saturno madureira
}

\begin{abstract}
RESUHO: 0 OBJETIVO DESTE ESTUDO FOI VERIFICAR A INFLUENCIA DA FADIGA MUSCULAR, DE MEMBROS SUPERIORES. NA ESTABILIDADE MANUAL DE SUJEI TOS DE AMBOS OS SEXOS ATRAVES DA REALIZAÇAO DE UMA TAREFA MOTORA. Foram sujeitos 20 alunos da universidade Federal de Santa Maria/RS, VOLUNTARIOS. DE AMBOS OS SEXOS ( $N=10$ MASC, E N= $10 \mathrm{FEM}$, ), A TAREFA FATIGANTE FOI REALIZADA NUM CICLOERGOMETRO ADAPTADO COM AUMENTO PRO GRESSIVO DE CARGAS E VELOCIDADE CONSTANTE DE 50 RPII, A ANALISE DE VÁ RIANCIA, TESTE de DUNCAN e teste "T" DE StUDENT REvelaram difERENCAAS SIGNIFICATIVAS ( $P<0.01 ; P<0.05 \mathrm{E} P<0.01$, RESPECTIVAMENTE) NOS RESULTADOS DAS VARAVEIS NUMERO DE ERROS E TEMPO DE PERMANENCIA EM ERRO DAS MAOS DIREITA E ESQUERDA, ENCONTROU-SE, TAMBEM DIFERENÇA SIGNIFICATIVA $(p<0,01)$ NA ESTABILIDADE MANUAL DA MAO DIREITA PARA AMBOS OS SEXOS, CUNCLUIU-SE GUE a FADIGA MUSCULAR INFLUENCIOU NA ES TABILIDADE MANUAL DOS SUJEITOS NA TAREFA MOTORA PROPOSTA,
\end{abstract}

ABSTRACT: THE PURPOSE OF THIS STUDY WAS TO VERIFY THE INFLUENCE OF MUSCULAR FATIGUE OF THE ARMS UPON MANUAL STABILITY ON A MOTOR TASK OF MALE AND FEMALE SUBJECTS, TWENTY VOLUNTARY STUDENTS (10 MALES AND 10 females) enrolled at Universidade Federal de Santa Maria/RS, served AS SUBJECTS. THE FATIGUE TASK WAS DEVELOPED THROUGH AN ADAPTED BYCICLE ERGOMETER INCREASING LOADS AND CONSTANT SPEED OF 50 RPM. ANALYS OF VARIANCE, DUNCAN'S TEST AND STUDENT T TEST SHOWED STATISTICAL DIFFERENCES $(p<0.01 ; p<0.05$ AND $P<0.01$, RESPECTIVELY) OF VARIABLES: NUMBER OF ERRORS AND PERMANENCE TIME IN ERROR OF RIGHT AND LEFT HANDS, ALSO, A SIGNIFICANT DIFFERENCE $(P<0,01)$ WAS FOUND ON THE RIGHT HAND MANUAL STABILITY FOR BOTH SEXES. WE CONCLUDE THAT MUSCULAR FATIGUE INFLUENCED HAND STABILITY OF ALL SUBJECTS AT THIS SPECIFIC MOTOR TASK.

* Professor da universidade estadual de Maringá/Pr.

* Professores da Universidade Federal de Santa Catarina/SC. 


\section{INTTRODUÇÃo}

A fadiga è um aspecto mutto difícil de ser estudado, principalmente porque ela assume um caráter tanto objetivo como subjetivo. A fadiga tanto pode ocorrer por sobrecarga de exercícios, afetando diretamente as grandes funções (sistema cárdio-respiratório), como pode ocorrer sensaçōes de fadiga sem exercícios precedentes. Outro fa tor que torna a fadiga um tema difícil de ser pesquisado é que cada indivíduo responde à mesma de diversas maneiras. Tanto um indivíduo motivado pode "ultrapassar" um padrão normal de fadiga, como outro individuo, por não estar motivado ou psicologicamente afetado por ou tros fatores, pode ficar aquém dos limites esperados.

Para ASTRAND (1980) muitos fisiologistas, nos últimos anos, têm debatido se a fadiga é de origem central ou periférica. Até hoje não se tem conhecimento se as várias conexões sinápticas podem entrar em fadiga. SIMONSON (1971) conclui que a transmissão da fadiga na juncão neuromuscular pode ser excluída. Entretanto, TAYLOR e STEFHENS (1972) evidenciaram em suas pesquisas que, em contração voluntária maxima, a fadiga da função neuromuscular é mais importante primeiro (durante o primeiro minuto), porém mais tarde, aumenta a fadiga do e lemento contrátil em vista da quantidade de substâncias transmissoras liberadas em cada transmissão de um impulso (apud ASTRAND, 1980).

A fadiga pode ser definida como um desequilibrio (homeostase al terada), podendo gerar sintomas físicos e mentais (CHRISTENSEN, apud ASTRAND , 1980). operacionalmente podemos dividíla, segundo LEVERO NI (1984), como a diminuição da capacidade de trabalho consecutiva à realização de uma tarefa.

Pesquisas mostram que índices fisiológicos da fadiga podem ser mensurados objetivamente por alguns fatores como aumento na instabilidade da coordenação neuromuscular, do ácido lático no sangue, do tem po de reação, do decréscimo de força, da glicose do sangue e do glicogènio muscular.

Muitos experimentos, nos anos 1960 e 1970, investigaram o papel da fadiga física no aprendizado das rabilidades motoras (BORG, 1981). A fadiga como elemento causador de padrões impróprios de ações motoras, de forma que os aprendizes praticassem movimentos errados, foi um dos temas investigados. Porém, poucos estudos têm atentado, mais 
profundamente neste tema, principalmente devido à ambiguidade do fenô meno.

Recentemente muitos estudos têm focalizado a relação entre a ati vidade fisiológica e a eficiência motora (BORG, 1981; BARTLEY, 1978 ; FLEURY et al11, 1981; FRANÇA et alii, 1985).

Estudo realizado por FRANÇA e outros (1985), para verificar o efeito da fadiga sobre a estabilidade manual na execução de uma tarefa motora, constatou que somente houveram diferenças significativas entre pré e pos teste $(\mathrm{p}<0.01)$ de indivíduos do sexo masculino, porém foram encontradas correlações significativas entre as variáveis do tes te de estabilidade (número de erros e tempo de permanência em erro) tanto no pré como no pós teste, para ambos os sexos.

Portanto, o objetivo do presente estudo fol verificar se a fadiga muscular de membros superiores influencia na estabilidade manual, procurando constatar o grau de Influência em sujeitos de ambos os sexos e na estabilidade da mão direita e da mão esquerda.

\section{MATERIAL E MÉTODO}

Participaram deste estudo 20 sujeitos (10 masculinos e 10 femin 1 . nos), voluntários, com idade variando entre 18 a 27 anos (20.65 2.03), estudantes do Curso de Educação Física da Universidade Federal de Santa Maria/RS.

Equipamentos utilizados:

Aparelho para o "Steadiness Test": uma placa de trabalho compos to de cinco testes, dos quais quatro foram encobertos. O teste de es tabilidade (Steadiness Test) foi realizado com quatro orifícios com diferentes diâmetros, escalonados em ordem decrescente. A urıdade de controle e registro foi acoplada a placa de trabalho fora das vistas do executante, fornecendo o nümero de erros das mãos direita (EMD) e esquerda (EME), bem como o tempo de permanência em errodestas (TPEME e TPEMD). Os estiletes foram conectados na placa de trabalho para serem manipulados com ambas as mãos simultâneamente.

Cicloergômetro: utilizou-se uma bicicleta da marca FUNBEC, na posiçāo invertida para ser operada com as mäos.

Fita cassete: foi utilizada uma fita gravada com o som do metrô 
nomo regulado com um batimento a cada 1.33 segundos.

Toca-fitas: um toca-fitas marca AIKO foi utilizado para determinar o ritmo de execução do teste de estabilidade.

Cronômetro: foram utilizados dois cronômetros digitais, centesimais, da marca CASIO QUARTZ.

Aparelho de Eletrocardiograma: utilizou-se o eletrocardiógrafo para a monitorização do individuo de acordo com as técnicas preconizadas por PINI (1983).

Procedimentos:

Preenchimento de uma ficha de identificação com dados pessoais.

Aplicação de eletrodos para monitorização da frequência cardiaca, seguindo as técnicas de monitorização para eletrocardiografia em esforço.

Tomada de frequência cardíaca de repouso com o sujeito sentado, imóvil, durante 5 minutos.

Teste de estabilidade manual (Steadiness Test): o individuo sen tou-se frontalmente a placa de trabalho, segurou os estiletes, um em cada mão, verticalmente a esta. Ao comando do avaliador de "atenção" e "jă", introduziu-se os dois estiletes ao mesmo tempo nos orifícios sem toca-los nas bordas, no fundo e sem apoiar as mãos na placa de trabalho. Esta introdução foi realizada seguindo a ordem do maior pa ra o menor orifício, permanecendo $3.99 \mathrm{seg}$. (3 batidas) em cada orifício e mantendo um intervalo, entre os orifícios, de $1.33 \mathrm{seg}$. 0 ritmo de execução de 3 tempos por orifício e o tempo de intervalo foi estabelecido pelo som do toca-fitas com a respectiva fita gravada ao som do metrônomo (observado por todos os sujeitos). o ava:iador sempre comunicava o final do teste aos sujeitos.

Tarefa motora fatigante de membros superiores de carga progressiva: o indivíduo monitorizado sentou-se numa cadeira (posição cômoda) frente ao cicloergömetro em posição invertida, iniciando a tarefa de manivelar com os membros superiores, a uma carga inicial de ze ro watts e velocidade constante de 50 RPM. A carga inicial foi mantida durante os 2 minutos iniciais, após a carga passou para 25 watts, aumentando-se progressivamente, de 2 em 2 minutos, com uma velocida- 
de constante de $50 \mathrm{RPM}$, até que o indivíduo atingisse a fadiga total dos membros superiores, estabelecida quando este não conseguisse man ter a velocidade acima de 30 RPM.

A fadiga cardíaca de cada sujelto fol registrada sempre nos últimos segundos antes da troca da carga de trabalho. o avaliador incentivava o sujeito a manivelar o máximo possível quando este estava diminuindo a velocidade do cicloergômetro. Em seguida, após o indivíduo ter atingido a fadiga, era realizado novamente o teste de esta bilidade manual no aparelho apropriado, instalado ao lado do cicloer gômetro. A frequência cardíaca de cada sujeito, após o exercício, fo1 registrada de 15 em 15 segundos durante 5 minutos.

\section{REsultados E DIscussão}

As Figuras 1 e 2 apresentam graficamente as médias $(\overline{\bar{X}})$ e os des vios padrões (s) dos escores do teste de estabilidade manual do sexo feminino e masculino, respectivamente.

Os resultados diferiram significativamente entre o pré e o pós teste para ambos os grupos (sexos), demonstrando um aumento significativo no número e tempo de permanência no erro, conforme resultados verificados através da análise de variância nas Tabelas 1 e 2; teste de Duncan nas Tabelas 3 e 4 e teste " $t$ ", de Student, nas Tabelas 5 e 6 .

Tabela 1 - Resumo da análisle da variância para a variável numero de erros.

\begin{tabular}{lrrr}
\hline Fonte de Variaçao & Media dos Quadrados & Valor R & Probabilidade F \\
\hline Mão & 8.450000 & 0.53698 & 0.5272 \\
Sexo & 26.450000 & 1.68085 & 0.1961 \\
Teste & 540.800000 & 34.36681 & 0.0001 \\
Resíduo & 15.736111 & & \\
& & & \\
& & &
\end{tabular}


FIgURA 1 - Teste de Estabilidade Manual da turma feminina no pré e pós-teste (média e desvio padrão)

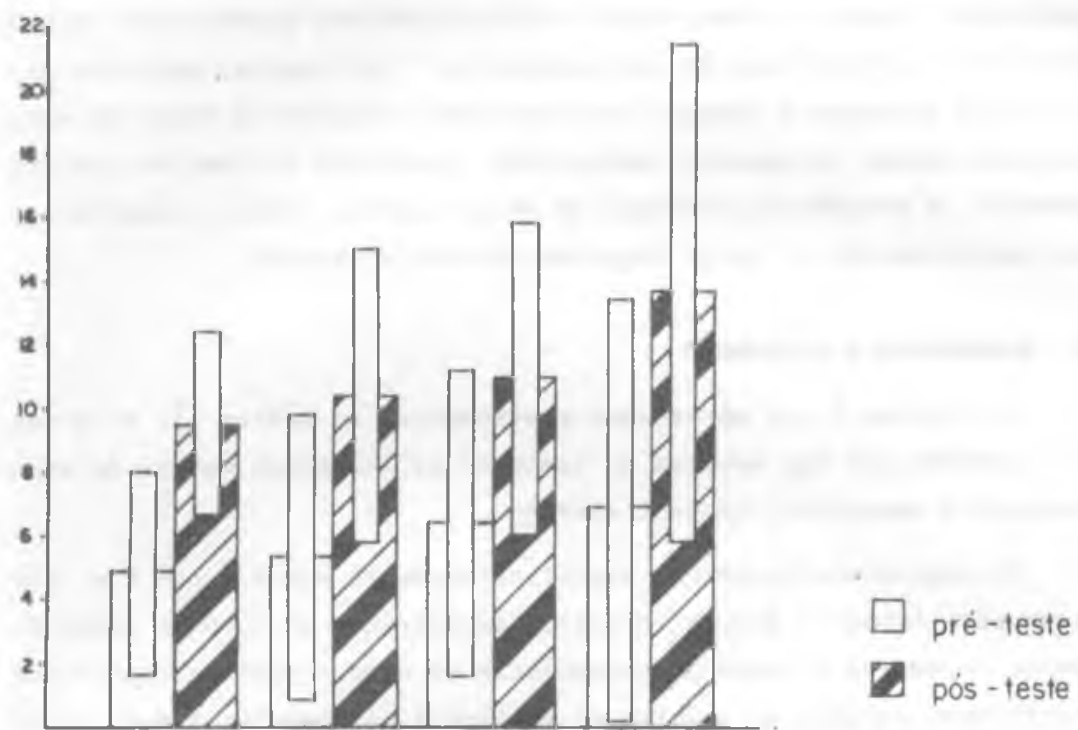

EMD = Nümero de erros da māo direita

TPEMD = Tempo de permanência em erro da mão direita

$\mathrm{EME}$ = Número de erros da mão esquerda

TPEMD = Tempo de permanência em erro da mão esquerda 


\section{FIGURA 2 - Teste de Estabilidade Manual da turma masculina no pré e pós-teste (média e desvio padrão)}

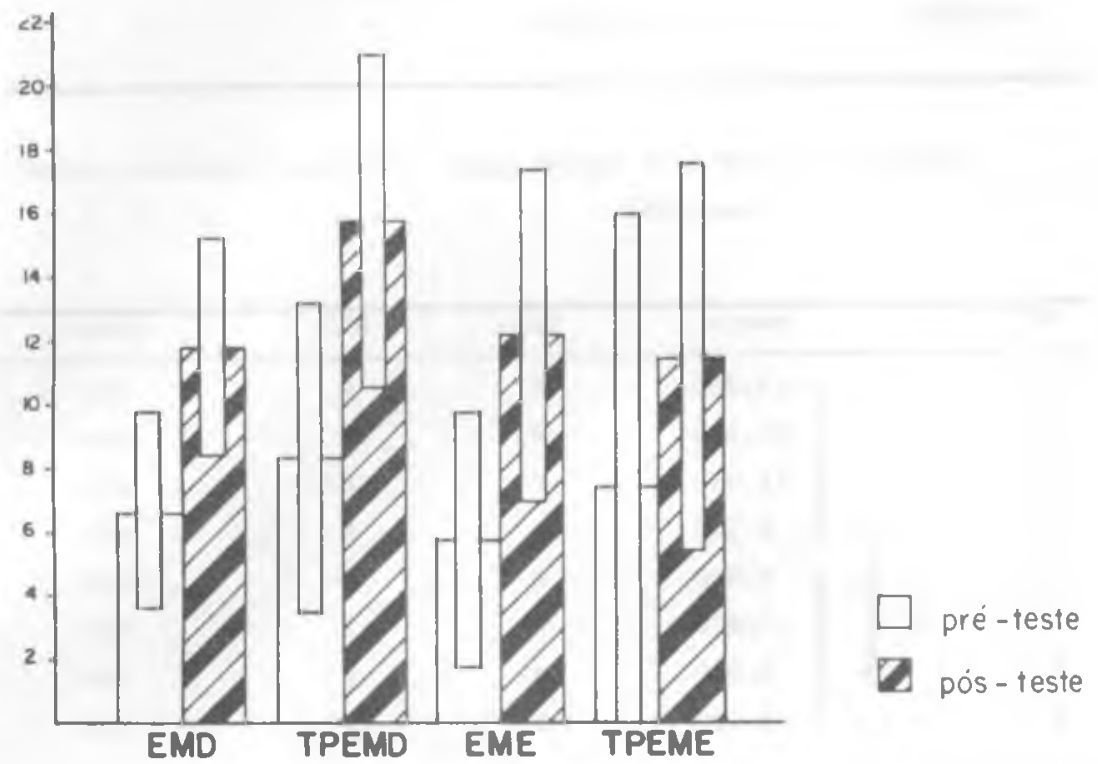

$E M D=$ Número de erros da mão direita

TPEMD = Tempo de permanência em erro da mão direita

$E M E$ = Nümero de erros da mão esquerda

TPEME = Tempo de permanência em erro da mão esquerda 
Tabela 2 - Resumo da análise de variância para a variável tempo de permanência em erro.

\begin{tabular}{crrc}
\hline Fonte de Variaçao & Media dos Quadrados & Valor F & Probabilidade \\
\hline Máo & 3.612500 & 0.09322 & 0.7589 \\
Sexo & 94.612500 & 2.44153 & 0.1186 \\
Teste & 812.812500 & 20.97505 & 0.0001 \\
Resíduo & 38.751389 & & \\
& & & \\
\hline
\end{tabular}

Tabela 3 - Teste de Duncan para a variavel numero de erros $(p<0.05)$

\begin{tabular}{|c|c|c|c|c|}
\hline No & Media & Sexo & Mao & Teste \\
\hline 1 & {$[12.200$} & M & E & Pós \\
\hline 2 & 11.800 & M & D & Pós \\
\hline 3 & 11.000 & F & $\mathrm{E}$ & Pós \\
\hline 4 & $L \quad 9.500$ & F & D & Pós \\
\hline 5 & {$[6.600$} & M & D & Pré \\
\hline 6 & 6.400 & F & E & Pré \\
\hline 7 & 5.800 & M & $\mathrm{E}$ & Pré \\
\hline 8 & {$[\quad 4,900$} & F & D & Pré \\
\hline
\end{tabular}

Tabela 4 - Teste de Duncan para a variável tempo de permanência em erro $(p<0.05)$

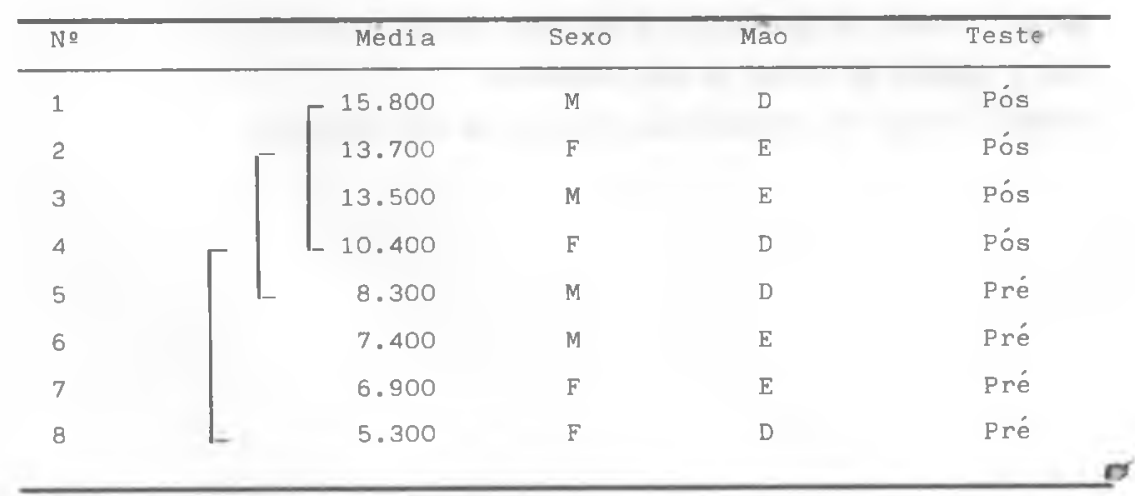


Tabela 5 - Valores médios e teste "t", de Student, para a variável número de erros entre pré e pós-teste na mão direita e esquerda - ambos os sexos

\begin{tabular}{cccccccc}
\hline Sexo & N & Mao & GL & Pre & Pos & tc & NS \\
\hline F & 10 & D & 9 & $4.9 \pm 2.33$ & $9.5 \pm 2.79$ & 5.71 & $0.01^{*}$ \\
F & 10 & E & 9 & $6.4 \pm 4.76$ & $11.0 \pm 4.94$ & 3.26 & $0.01^{*}$ \\
M & 10 & D & 9 & $6.6 \pm 2.83$ & $11.8 \pm 3.45$ & 4.62 & $0.01 *$ \\
M & 10 & E & 9 & $5.8 \pm 4.10$ & $12.2 \pm 5.34$ & 4.70 & $0.01 *$ \\
\hline
\end{tabular}

* $t=3.25$

Tabela 6 - Valores médios e teste "t", de Student, para a variável tempo de permanência em erro entre pré e pós-teste na mão direita e esquerda - ambos os sexos

\begin{tabular}{cccccccc}
\hline Sexo & N & Mao & GL & Pre & Pos & tc & NS \\
\hline F & 10 & D & 9 & $5.3 \pm 4.05$ & $10.4 \pm 4.69$ & 5.01 & $0.01^{*}$ \\
F & 10 & E & 9 & $6.9 \pm 6.64$ & $13.7 \pm 7.80$ & 4.06 & $0.01^{*}$ \\
M & 10 & D & 9 & $8.3 \pm 4.92$ & $15.8 \pm 5.24$ & 3.75 & $0.01^{*}$ \\
M & 10 & E & 9 & $7.4 \pm 8.74$ & $13.5 \pm 6.18$ & 2.91 & $0.05^{*}$ \\
& & & & & & & \\
\hline$t=3.25$ & $*$ & $t=2.26$
\end{tabular}

Para analisarmos a homogeneidade dos escores, dentro dos testes, comparamos nas figuras 3 e 4 os resultados dos grupos' (sexos) em prè e pós-teste, respectivamente. Através dos resultados da análise de variância dos testes (Tabelas 7, 8, 9 e 10), podemos constatar que houveram diferenças significativas no pré e no pós-teste em relação as variáveis sexo e mão dos sujeitos, confirmando assim que os resul. tados apresentaram um certo grau de homogeneidade nos testes. 
FIGURA 3 - Teste de Estabilidade Manual da turma feminina $x$ turma masculina no pré-teste (média e desvio padrão)

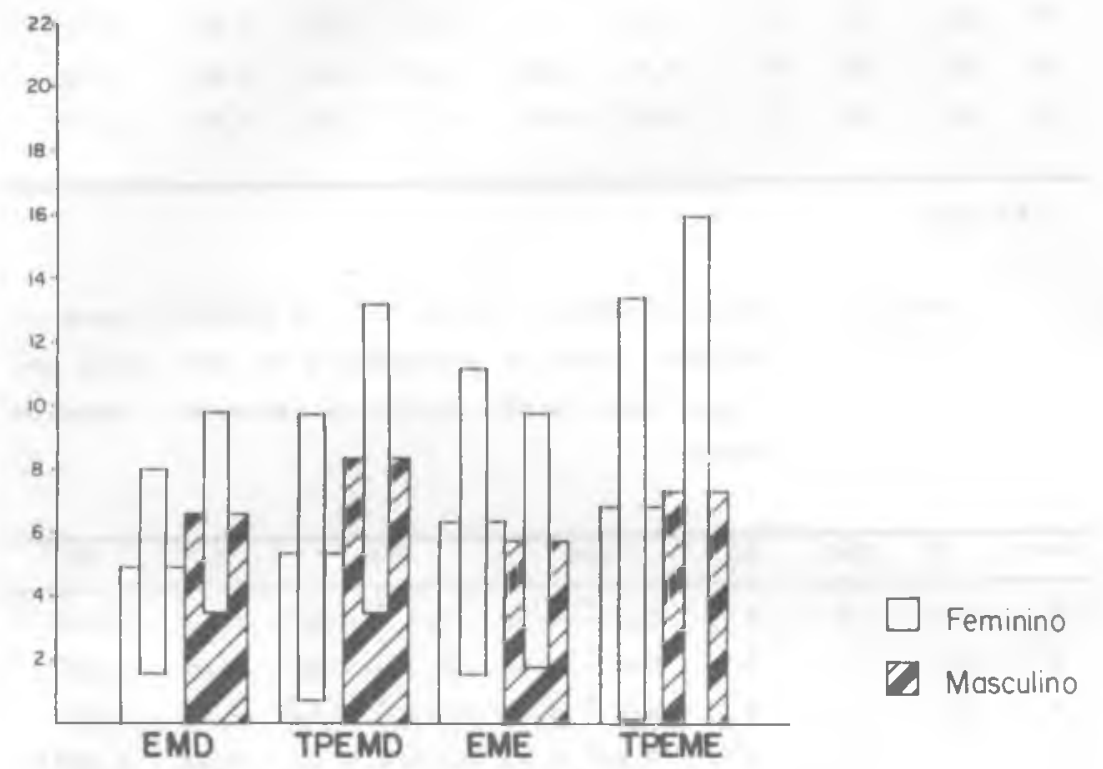

$E M D=$ Número de erros da mão direita

TPEMD = Tempo de permanência em erro da mão direita

$\mathrm{EME}=$ Numero de erros da mão esquerda

TPEME = Tempo de permanência em erro da mão esquerda 
FIGURA 4 - Teste de Estabilidade Manual da turma feminina $x$ turma masculina no pós-teste (média e desvio padrão)

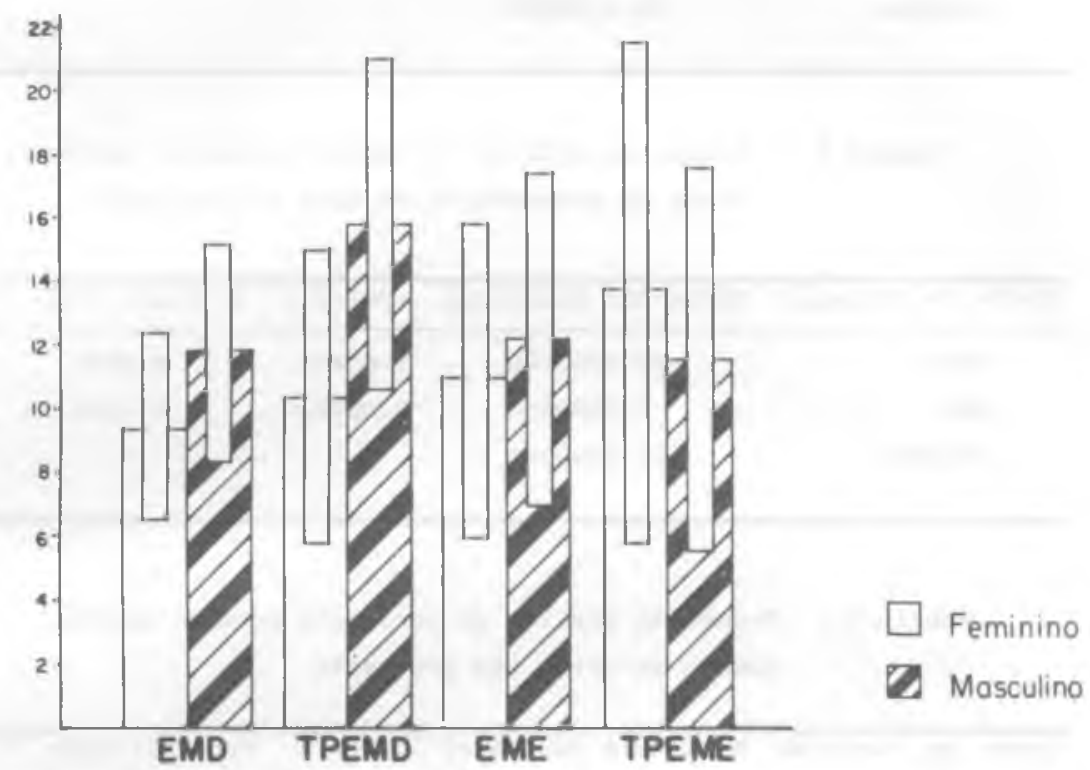

EMD = Número de erros da mão direita

TPEMD = Tempo de permanência em erro da mão direita

$E M E$ = Nümero de erros da mão esquerda

TPEME = Tempo de permanência em erro da mão esquerda 
Tabela 7 - Resumo da análise de variância para a variável número de erros no pós-teste

\begin{tabular}{crcc}
\hline Fonte de Variaçåo & Media dos Quadrados & Valor F & Probabilidade \\
\hline Sexo & 30.6250000 & 1.68141 & 0.2004 \\
Mão & 9.0250000 & 0.49550 & 0.5072 \\
Resíduo & 18.2138889 & & \\
& & & \\
\hline
\end{tabular}

Tabela 8 - Resumo da análise de variância para a variável tempo de permanência em erro no pós-teste

\begin{tabular}{|c|c|c|c|}
\hline Fonte de Variaçao & Media dos Quadrados & Valor F & Probabilidade \\
\hline Sexo & 30.6000000 & 1.81801 & 0.1830 \\
\hline Mão & 2.5000000 & 0.06723 & 0.7926 \\
\hline Resíduo & 37.1833333 & 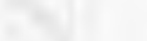 & \\
\hline
\end{tabular}

Tabela 9 - Resumo da análise de variāncia para a variável número de erros nos pré-teste

\begin{tabular}{crcc}
\hline Fonte de Variaçao & Media dos Quadrados & Valor F & Probabilidade F \\
\hline Sexo & 3.0250000 & 0.22816 & 0.6408 \\
Mão & 1.2250000 & 0.09239 & 0.7605 \\
Resíduo & 13.2583333 & & \\
& & & \\
\hline
\end{tabular}

Tabela 10 - Resumo da análise de variâncla para a variável tempo de permanência em erro no pré-teste

\begin{tabular}{crccc}
\hline Fonte de Variaçao & Media dos Quadrados & Valor F & Probabilidade & F \\
\hline Sexo & 30.6250000 & 0.75956 & 0.6067 \\
Mão & 1.2250000 & 0.03038 & 0.8568 \\
Residuo & 40.3194444 & &
\end{tabular}


Os resultados da análise de variância nas variáveis número de erros e tempo de permanência em erro, quando separados somente em gru pos (sexos) não apresentaram diferenças significativas.

Para constatar se houveram diferenças significativas na estabilidade (número de erros e tempo de permanência em erro) nas mãos direita e esquerda para os grupos e testes, realizamos a análise de va riância para os escores separados (mão direita e mão esquerda). Os resultados são apresentados nas Tabelas 11, 12, 13 e 14. Podemos ve rificar que houveram diferenças significativas nos escores de ambas as mãos nas variáveis numero de erros e tempo de permanência em erro nos testes, confirmando ainda mais os resultados anteriores. Porém, constatou-se que houveram diferengas significativas nos escores das variáveis número de erros e tempo de permanência em erro nos grupos (sexo) somente para a mão direita, fato que não ocorreu com os resul tados da mão esquerda.

Tabela 11 - Resumo da análise de variâncla para a variável número de erros da mão direita

\begin{tabular}{lrrrr}
\hline Fontes de Variaçao & Media dos Quadrados & Valor F & Probabilidade & F \\
\hline Sexo & 40.000000 & 4.80962 & 0.0328 \\
Testes & 240.100000 & 28.86974 & 0.0001 \\
Residuo & 8.316667 & & \\
\hline
\end{tabular}

Tabela 12 - Resumo da análise de variância para a variável tempo de permanência no erro da não direita

\begin{tabular}{lrrr}
\hline Fontes de Variação & Média dos Quadrados & Valor F & Probabilidade \\
\hline Sexo & 176.400000 & 4.81876 & 0.0081 \\
Testes & 396.900000 & 17.59222 & 0.0004 \\
Residuo & 22.561111 & & \\
\hline
\end{tabular}


Tabela 13 - Resumo da análise de variância para a variável número de erros da mão esquerda

\begin{tabular}{lrrrr}
\hline Fonte de Variaçao & Media dos Quadrados & Valor F & Probabilidade F \\
\hline Sexo & 0.900000 & 0.03887 & 0.8390 \\
Teste & 302.500000 & 13.06382 & 0.0012 \\
Resíduo & 23.155555 & & \\
\hline
\end{tabular}

Tabela 14 - Resumo da análise de variância para a variável tempo de permanência no erro da mão esquerda

\begin{tabular}{lrrr}
\hline Fonte de Variaçáo & Media dos Quadrados & Valor F & Probabilidade \\
\hline Sexo & 0.22500 & 0.00410 & 0.9479 \\
Testes & 416.02500 & 7.57212 & 0.0090 \\
Resíduo & 54.94160 & & \\
\hline
\end{tabular}

A Tabela 15 apresenta numericamente as médias e desvios padrões das frequências cardiacas no exercício e período de recuperação de am bos os grupos (feminino e masculino)

Tabela 15 - Medias e desvios padrões das frequências cardiacas

\begin{tabular}{|c|c|c|c|c|c|c|c|}
\hline \multicolumn{4}{|c|}{ EXERCİCIOS } & \multicolumn{4}{|c|}{ RECUPERAÇÃO } \\
\hline Turma & Idade & $\begin{array}{c}\mathrm{FC} \\
\text { Inicial }\end{array}$ & $\begin{array}{c}\mathrm{FC} \\
\text { Final }\end{array}$ & $\begin{array}{l}\text { Tempo p/ } \\
\text { fatigar }\end{array}$ & $\begin{array}{l}\text { Maxima } \\
\text { Carga }\end{array}$ & $\begin{array}{c}\text { FC } \\
\text { Inicial }\end{array}$ & $\begin{array}{c}\mathrm{FC} \\
\text { Final }\end{array}$ \\
\hline \multirow{2}{*}{ FFIM } & 20 & 79.4 & 167.1 & 10.2 & 105 & 258.5 & 96.6 \\
\hline & \pm 2.6 & \pm 10.4 & \pm 12.9 & \pm 1.0 & \pm 10.5 & \pm 15.0 & \pm 16.4 \\
\hline \multirow[t]{2}{*}{ MAS } & 20 & 70.6 & 168.0 & 12.7 & 147.5 & 145.3 & 90.2 \\
\hline & \pm 0.8 & \pm 8.9 & \pm 21.26 & \pm 0.7 & \pm 7.9 & \pm 26.0 & \pm 16.4 \\
\hline
\end{tabular}

Para uma melhor visualização dos resultados acima, nas Figuras 5 e 6 estão plotados, graficamente, o comportamento da frequéncia cardíaca durante o exercício e período de recuperação dos grupos femi nino e masculino (sexo). 
FIGURA 5 - Comportamento da frequência cardíaca do grupo feminino durante o exercício e recuperação

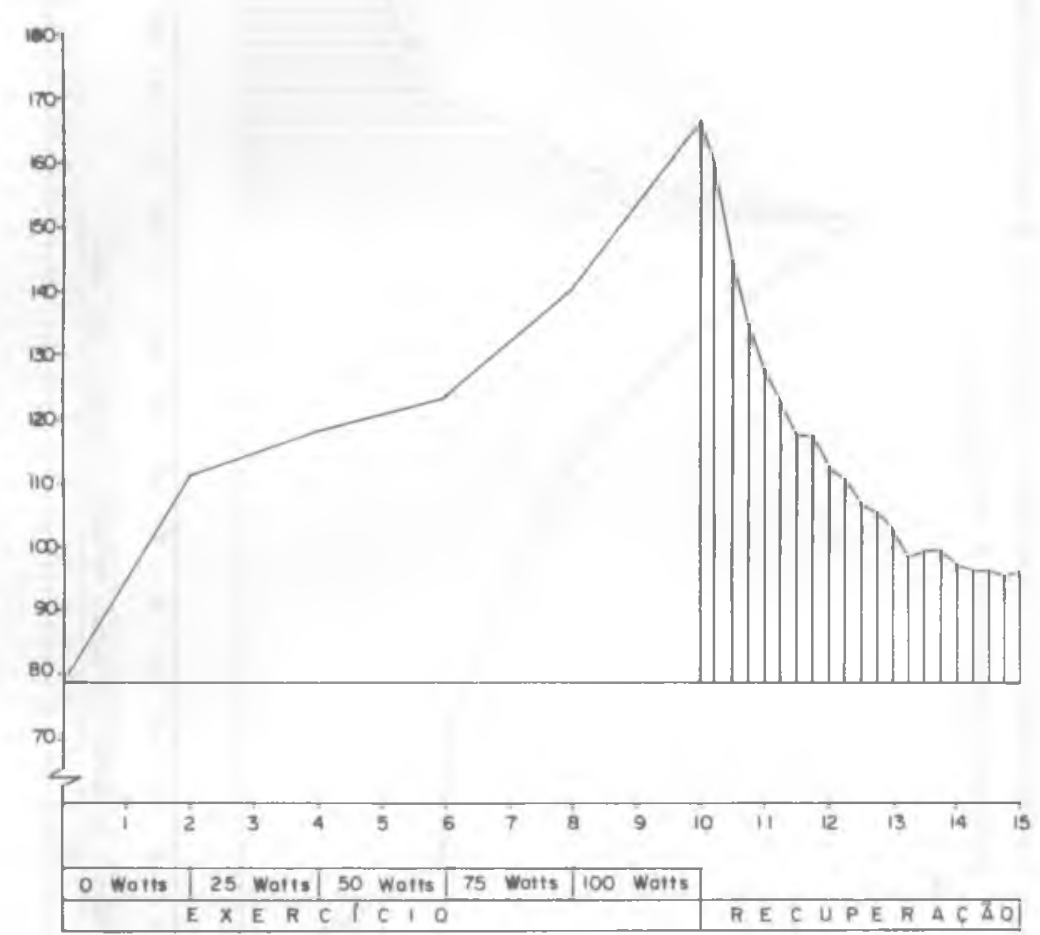


FIGURA 6 - Comportamento da frequencia cardiaca do grupo masculino durante o exercício e recuperação

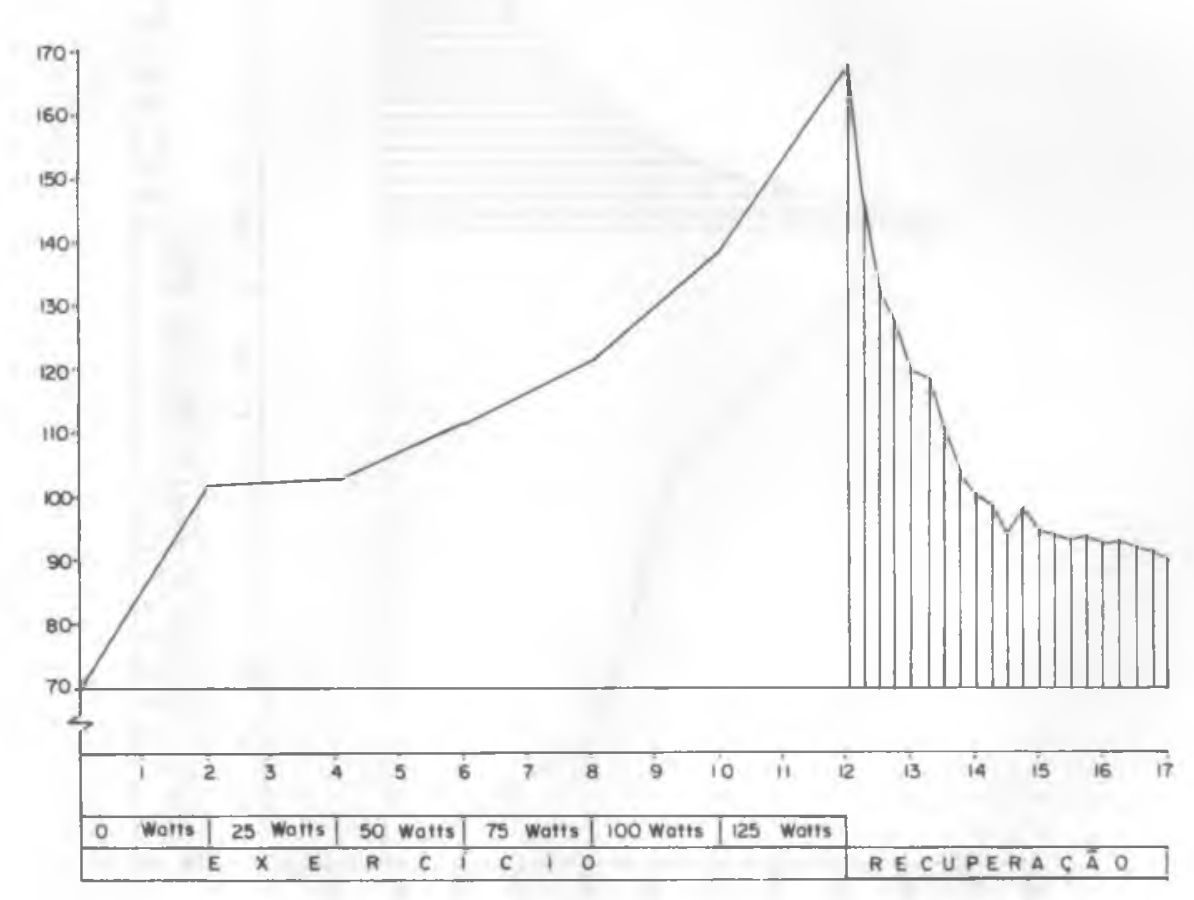

3

$\therefore$ 
Alguns fisiologistas confirmam que a fadiga influenciano decrés cimo da performance ou capacidade de trabalho, devido as mudanças bioquímicas no tecido muscular. Muitas pesquisas têm sido feitas pa ra constatar a influência da fadiga nas tarefas motoras. BORG (1969), apud BORG (1981), estudou o comportamento da estabilidade manual de sujeitos submetidos a três cargas de trabalho no cicloergönetro (25, 55 e $85 \%$ de sua carga máxima) durante 20 segundos, imediatamente após o término de cada carga. Verificou que a estabilidade era ótima durante o período de repoúso e piorou com o aumento da carga de trabalho. Estudos realizados por GUTIN, FOGLE, MEYER e JAEGER (1974), apud BORG (1981), utilizando três cargas de trabalho induzidas pelas frequências cardíacas de 100,130 e 160 batimentos por minuto, em ca da sujeito, constataram que a estabilidade foi marcadamente afetada pelo exercicio anterior e a instabilidade foi maior após o exercício de frequência de 160 batimentos por minuto. Podemos inferir em nosso estudo que utilizou o exercício físico com aumento progressivo da carga como meio de variação sistemática do nível de ativação (fadiga), que a estabilidade manual foi afetada significativamente após o exer cício, ou seja, a tarefa fatigante provocou a instabilidade manual dos sujeitos.

Para HANSON E LOFTHUS (1978), apud LOFTHUS e HANSON (1980), os efeitos da fadiga são mais prejudiciais aos membros não preferidos. As performances das tarefas que requerem grande precisão, força ou controle motor resultam num maior decréscimo da performance após a fadiga. Isto talvez pudesse explicar a diferença encontrada nos escores da estabilidade das mãos dos sujeitos. Porém, devido ao fato de não termos realizado anteriormente testes de lateralidade com os sujeitos, para verificar qual a mão preferida, não podemos afirmar que a fadiga influenciou os membros não preferidos. Este assunto po derá ser estudado posteriormente em outros trabalhos.

Muitos pesquisadores nesta área da fadiga têm utilizado como crí tério pré-deteminante deste estado fatores como: a frequência cardíaca de 180 batimentos por minuto desenvolvida através de um aumento da resistência na bicicleta ergométrica (BENSON,1968; CARRON,1972); a exaustão expressada pelo término do trabalho num teste máximo de banco ou num teste de manivelar com os braços (ALDERMAN, 1965; CLARK \& STEVENSON, 1962; MALOMSOKI \& SZMODIS, 1970; SORGE, 1960 ; VLAHOV, 
1977), a fixação de carga na bicicleta ergométrica (BLITZ \& VAN MOORST, 1978; BORG, 1978; MALCNSOKI \& SZMODIS, 1970); horas de trabalho para tarefas especificas como: dirigir, correr, etc. (BROWN, 1967; CASE et alii, 1965); um decréscimo no trabalho trazido pela máxima contração voluntária (HEYES, 1975; KROLL, 1973, apud FLEURY et alii, 1981). KAR POVICH ,1963 (-apud BARTLEY, 1965) e LEVERONI (1984) afirmam que fatos como: temperatura, umidade, hora do dia, altura e tipo de trabalho, en tre outros, podem acelerar a instalação da fadiga. Assim, existem tam bém fatores que retardam o aparecimento da fadiga como: motivação, eficiência mecânica e nível de treinamento.

Para STEGEMAN (1979) a fadiga muscular è causada por desequilíbrio fisico-químico, onde ocorre um esgotamento da energia local depois de um tempo determinado. Ele utilizou como medida para a fadiga muscular o valor do pulso de recuperação. Nos trabalhos não-fatigantes, a frequência cardíaca atinge seu valor de repouso em 2-3 minutos e segue graficamente uma função exponencial negativa simples. No caso de um trabalho fatigante a função exponencial é, no mínimo, a soma de duas funções exponenciais e o tempo para retornar à frequência de repouso pode durar horas.

Percebeu-se que a tarefa proposta por este estudo foi considerada fatigante, por seguirmos os estudos de alguns fisiologistas que afirmam que uma tarefa pode ser considerada fatigante quando: a exaustão é expressada pelo término do trabalho no teste de manivelar com OS braços (ALDERMAN, 1965; CLARK \& STEVENSON, 1962; MALONSOKI \& SZMODIS, 1970; SORGE, 1960; VLAHOV, 1977 (apud FLEURY et alii, 1981); os valores de pulso na recuperação não atingem seu valor de repouso em 2 ou 3 minutos, e também seguem graficamente a soma de duas funçõe. expo nenciais (STEGEMAN, 1979).

\section{CORCLUSŌES E SUGESTIÕES}

Os resultados obtidos neste estudo nos levam a crer que a aprendizagem (gesto técnico desportivo) poderá não ocorrer se os indivíduos estiverem fatigados pois não terão, provavelmente, estabilidade su ficiente nos membros superiores para realizá-lo. Daí a importância de se seguir uma sequencia correta com relaçāo a ordem das tarefas nu ma sessão de atividades físicas. 
Os resultados também mostraram evidências de que em escolas onde as aulas de Educação Fĩsica são entremeadas com outras disciplinas, tais como: Matemática, Fïsica, Desenho e outras, os alunos ao retornarem às salas de aula não conseguem estabilidade manual suficiente por estarem com os membros superiores fatigados, prejudicando o rendi mento do aluno.

Dentro do que foi exposto anteriormente e de acordo com os resul tados obtidos, as seguintes conclusões parecem ser garantidas:

- a fadiga muscular, de membros superiores, influenciou na esta bilidade manual para sujeitos de ambos os sexos;

- a estabilidade manual foi significativamente diferente entre os sexos, somente nos resultados da mão direita;

Acreditamos ser de grande valia a repetição desta pesquisa utili zando-se, entretanto, a mão preferida para a execução de tarefas motoras simples. Esta sugestão está ligada ao fato de, no presente estudo, termos constatado diferença significativa nos escores da mão di reita para os sujeitos de ambos os sexos, não ocorrendo o mesmo com a mão esquerda.

Sugerimos, ainda, que outros estudos sejam realizados com a fina lidade de verificar porque os escores da mão esquerda não são signifi cativos.

\section{REFFRÊNCIAS BIBLIOGRÁFICAS}

1 ASTRAND, Per Olof. Tratado de fisiologia do exercício. 2 ed., Rio de Janeiro, Interamericana, 1980.

2 Bartley, S. Howard. Fatigue. Perceptual and Motor Skills, (53): 958,1981 .

3 . Fatigue: Mechanis sms and management. Springfield, Illinois, Charles C. Thomas Pub., 1965.

4 BLITZ, Peter S. \& VAN MOORST, Annemiek. Physical fatigue and the perception of differences in load: a signal detection approach. Perceptual and Motor Skil1s, (46):779-790, 1978. 
5 BORG, Gunnar \& SJOBERG, Hans. The variation of hand steadiness with physical stress. Journal of Motor Behavior, 13(2):110-16, 1981 .

6 DWYER, Joffrey. Influence of physical fatigue on motor performan ce and learning. Hesearch Quarterly, 32(1):3-11, 1972.

7 FLEURY, Michelie et alii. Influence of different: types of physi. cal fatigue on a visual detection task. Perceptual and Motor Skills, (53):723-30, 1981 .

8 FOX, Edward L. \& MATHEWS, K. Bases Fisiológicas da Educação Físi ca e dos Desportos. 3 ed., Rio de Janeiro, Interamericana, 1983.

9 FRANÇA, Nanci et alii. Efeito da fadiga sobre a estabilidade manual na execução de uma tarefa motora. Revista Brasileira de Ciência do Esporte, $\underline{7}(1): 22,1985$.

10 LeVeroni, A. F. Fisiologia do Exercício. 5 ed., Rio de Janeiro, Guanabara Koogan, 1984 .

11 LOFTHUS, Geraldine K. \& HANSON, Cheryl. The influence of laterality and fatigue upon the performance of a two-handed reaction task. Research Quarterly, $\underline{51}(3): 501-8,1980$.

12 MAGILL, R. A. Aprendizagem Motora: conceitos e aplicações. São Paulo, Edgard Blucher, 1984.

13 PINI, Mário Carvalho. Fisiologia Esportiva. 2 ed., Rio de Janeiro, Guanabara Koogan, 1983.

14 STEGEMANN, Jurgen. Fisiologia do Esforço. 2 ed., Rio de Janeiro, Cultura Médica, 1979.

ESTE TRABALHO FOI REALIZADO NO LABORATÓRIO DE PESQUISA E ENSINO DO MOVIMENTO HUMANO (IAPEM) DO CENTRO DE EDUCAÇÃO FÍSICA E DES PORTOS/UFSM. 